\title{
Multiband Negative Permittivity Metamaterials and Absorbers
}

\author{
Yiran Tian, Guangjun Wen, and Yongjun Huang \\ Key Laboratory of Broadband Optical Fiber Transmission and Communication Networks, Ministry of Education, \\ School of Communication and Information Engineering, University of Electronic Science and Technology of China, \\ Chengdu 611731, China
}

Correspondence should be addressed to Yongjun Huang; yongjunh@uestc.edu.cn

Received 30 March 2013; Revised 4 July 2013; Accepted 10 July 2013

Academic Editor: Armin Gerhard Aberle

Copyright (C) 2013 Yiran Tian et al. This is an open access article distributed under the Creative Commons Attribution License, which permits unrestricted use, distribution, and reproduction in any medium, provided the original work is properly cited.

Design and characteristics of multiband negative permittivity metamaterial and its absorber configuration are presented in this paper. The proposed multiband metamaterial is composed of a novel multibranch resonator which can possess four electric resonance frequencies. It is shown that, by controlling the length of the main branches of such resonator, the resonant frequencies and corresponding absorbing bands of metamaterial absorber can be shifted in a large frequency band.

\section{Introduction}

Metamaterials, defined as artificial structures not found in nature, possess interesting properties, for example, negative refraction, perfect image, backward-wave radiation, reversals of both Doppler shift and Cherenkov radiation, and so forth [1-4]. It has recently shown that metamaterials can be used in various areas including microwave and optical components, absorbers, invisible cloaks, and so forth [5-9]. Due to these exciting properties and applications of metamaterials, in the past decade, various types of metamaterial configurations have been reported, operating at very wide frequency spectra ranged from microwave, $\mathrm{THz}$, and even optical frequencies [10-13].

Quite recently, dual-band, multiband, and even band tunable metamaterials with single negative permeability or permittivity and double negative properties have been reported to enhance the operating frequency bands [1419]. Also, the band-enhanced metamaterial absorbers were designed as well by using multiband electric resonators [2023]. The mentioned band enhanced techniques for metamaterials and absorbers, however, restricted from their complex configurations and difficult controlling abilities.

On another hand, in our previous research results [7], we have experimentally and numerically demonstrated a snowflake-shaped metamaterial absorber and obtained a well agreement between the measured and simulated results.
The snowflake-shaped metamaterial resonator was composed of equal three main branches and six side branches. However, we found that it could achieve multiresonance frequencies when the main branches were not equal. In this paper, we firstly propose a simple design of negative permittivity metamaterial by using a multibranch resonator which can possess four electric resonance frequencies. The transmission and reflection characteristics are firstly investigated and then the effective electromagnetic parameters retrieved from the $S$-parameters are determined. The tuning effects of the four operating frequencies by altering the main branches are discussed. Due to the fact that most of the single negative permittivity metamaterials are used to design the metamaterial absorbers in such research area, the multiband metamaterial absorber constructed by the proposed resonators is also discussed in this paper.

\section{Model Design}

The unit cell of the proposed multiband negative permittivity metamaterial is presented in Figure 1. There are four mainbranches with different lengths that intercrossed each other and eight pair of subbranches with equal length $f=0.86 \mathrm{~mm}$ located on the main branches with angle of $60^{\circ}$ between the main and subbranches. To obtain four electronic resonance points, the proposed resonator should be at least $C_{2}$ 


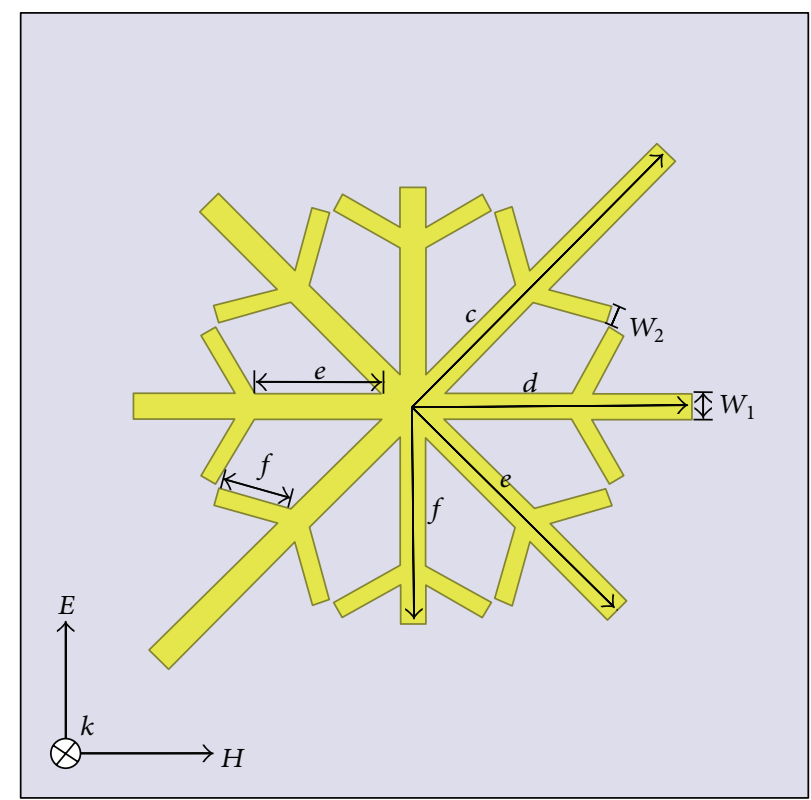

FIGURE 1: Unit cell presentation of the multiband negative permittivity metamaterial (color online).

symmetry to meet that there are two current routes with equal magnitudes and outphase flow orientations [24, 25]. The subbranches in the resonator are used to enhance the capacitance, resulting in the enhancement of electronic resonance strength, and also can reduce the size of resonator. The widths of the main and subbranches are $w_{1}=0.3 \mathrm{~mm}$ and $w_{2}=0.2 \mathrm{~mm}$, respectively, and the distance between the center of the resonator and the locations of subbranches is fixed as $e=1.45 \mathrm{~mm}$. The length of each main branch can be changed and will affect the operating frequencies, which will be discussed later.

We firstly demonstrate the multiresonance and transmission characteristics of the proposed negative permittivity metamaterials. For simply, the resonator with copper thickness of $0.017 \mathrm{~mm}$ is placed on a Rogers Ro4003 dielectric substrate with thickness of $1 \mathrm{~mm}$ (the relative permittivity is 3.55 and dielectric loss tangent is 0.0027 ). The other parameters of the resonator are defined as $a=4.1 \mathrm{~mm}, b=3.2 \mathrm{~mm}, c=$ $3.3 \mathrm{~mm}$, and $d=2.5 \mathrm{~mm}$, by using finite-element-methodbased commercial electromagnetic simulator (Ansoft HFSS V14). The period spaces between the resonators along the $E$ field and $H$-field are $9 \mathrm{~mm}$ (as shown in Figure 1).

\section{Simulation and Results}

For simulations, the unit cell is paced in a hollow waveguide with cross-section of $9 \times 9 \mathrm{~mm}^{2}$. The side walls of the waveguide perpendicular to the $E$-field are defined as perfect electric boundaries and those perpendicular to the $H$-field are defined as perfect magnetic boundaries to simulate an infinite period situation. The front and back planes parallel to the plane of the resonator are set as wave ports.

By using the previously mentioned dimensional parameters, we obtain the numerical transmission and reflection

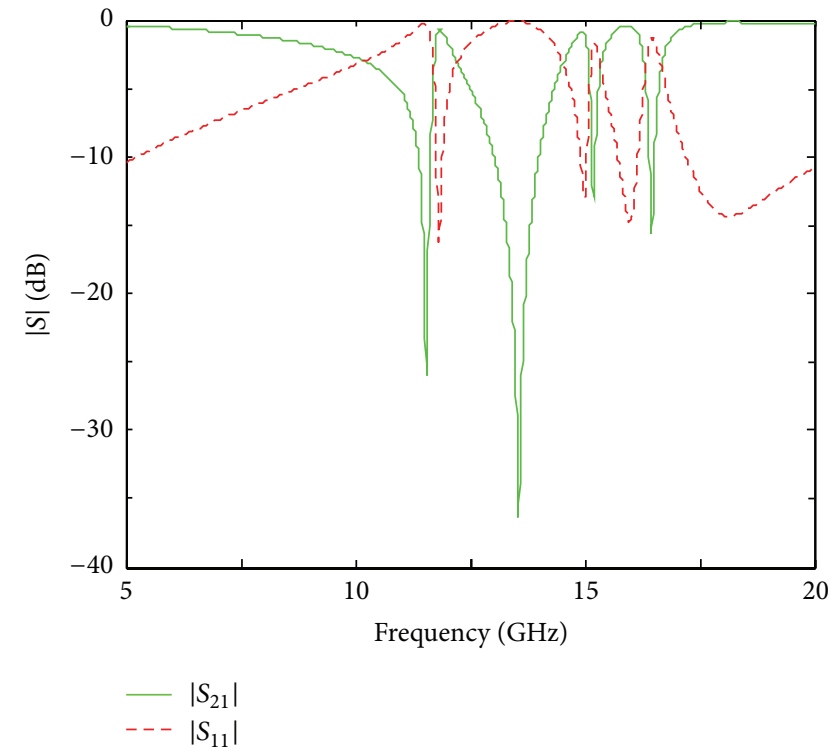

FIGURE 2: Simulated transmission and reflection results of the proposed multiband metamaterial (color online).

results of the proposed multibranch resonator by HFSS software, as show in Figure 2. It can be seen that there are clearly four transmission dips that appeared in the frequency range of $10-17.5 \mathrm{GHz}$; that is, four resonances are obtained at $11.55 \mathrm{GHz}, 13.58 \mathrm{GHz}, 15.22 \mathrm{GHz}$, and $16.82 \mathrm{GHz}$.

To demonstrate the negative permittivity properties, we retrieve the effective electromagnetic parameters from the simulated $S$-parameters by using the retrieving method mentioned by Smith et al. [26]. The results are illustrated in Figure 3, and it is shown that, near the reflection dips as presented in Figure 2, the multiband metamaterial possesses 


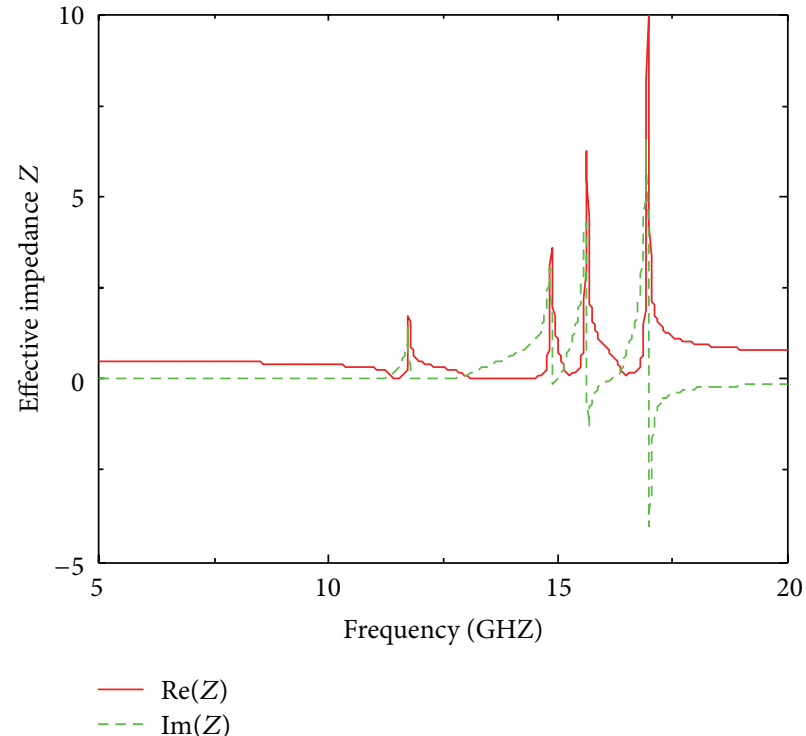

(a)

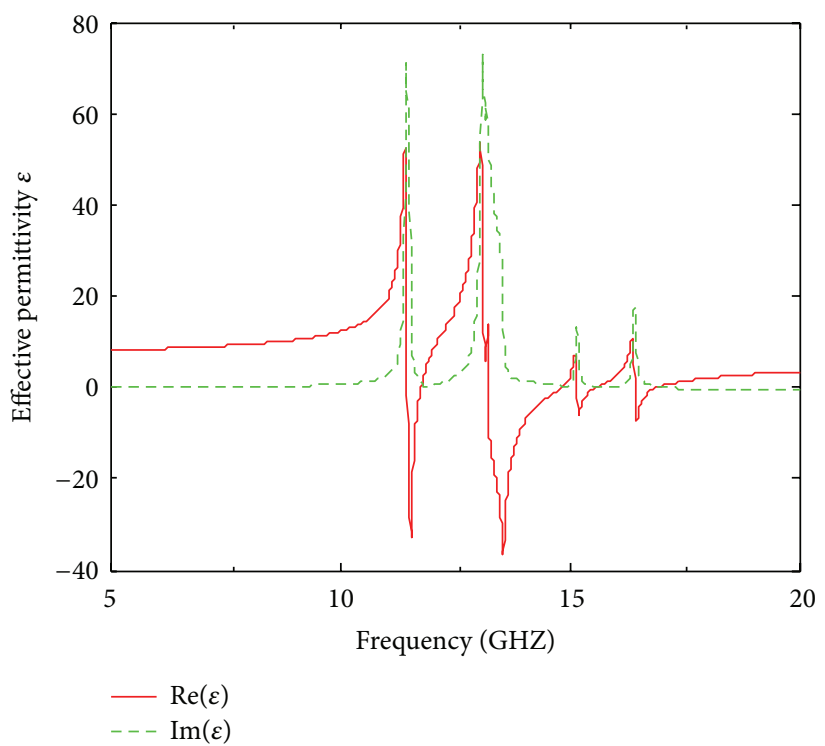

(c)

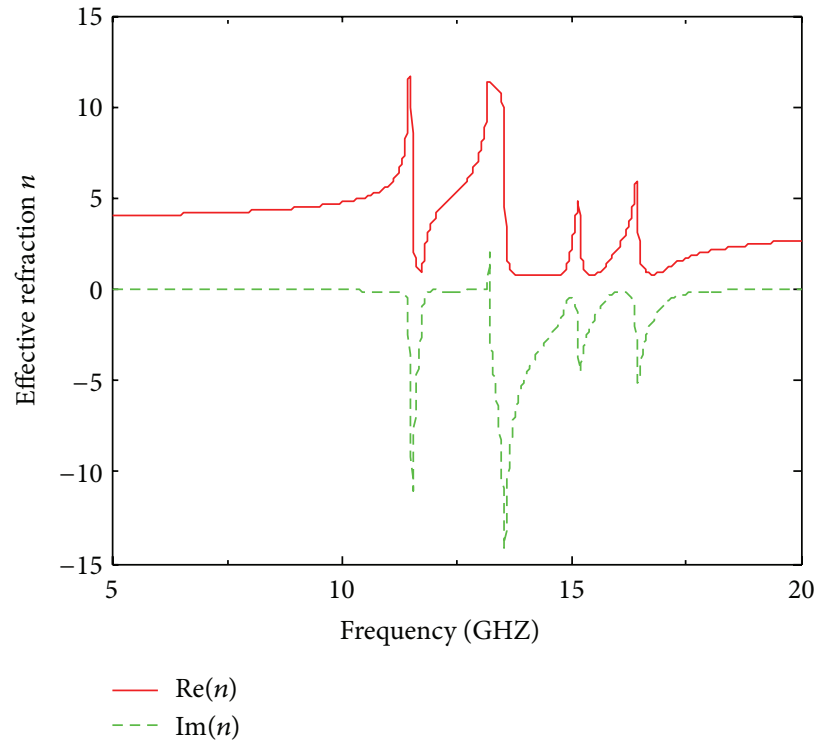

(b)

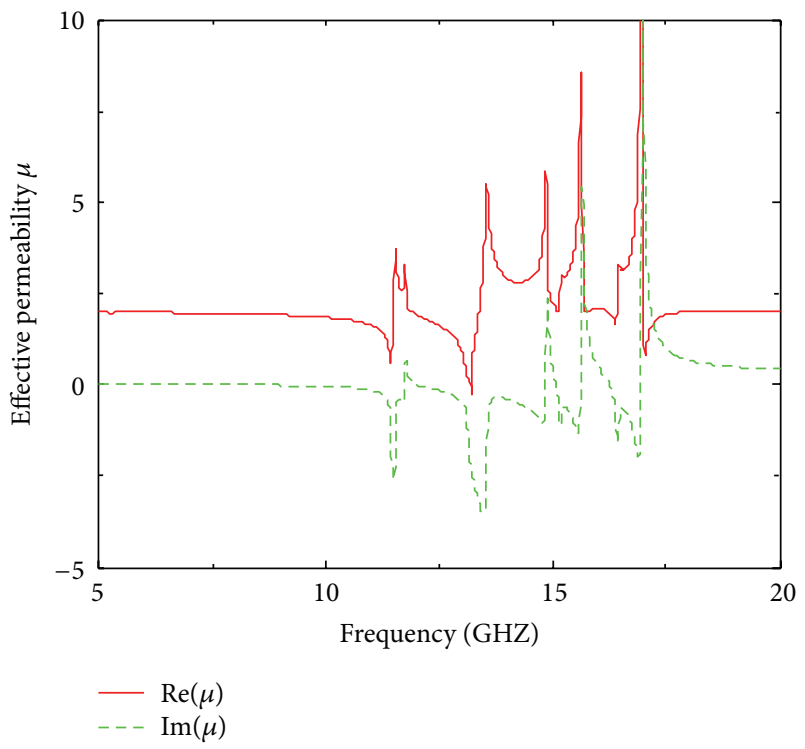

(d)

Figure 3: Retrieved effective electromagnetic parameters from the simulated $S$-parameters (color online).

high impedance characteristics (see Figure 3(a)), and the effective refractive index in the whole frequency band is positive (see Figure 3(b)). As shown in Figure 3(c), four typical electric resonances are obtained, and correspondingly four negative permittivity bands appeared at $11.55-11.75 \mathrm{GHz}$, $13.58-14.85 \mathrm{GHz}, \quad 15.22-15.62 \mathrm{GHz}$, and $16.82-16.96 \mathrm{GHz}$. Moreover, the retrieved effective permeability as shown in Figure 3(d) indicates that two antimagnetic resonances appear at first two resonance frequencies, and contrarily there are two typical magnetic resonances for the last two resonant frequencies, but all of them cannot lead to the negative permeability properties. This is due to the fact that, in the last two resonant frequencies, the bianisotropy properties occurred [27].

\section{Discussions}

The advantage of the proposed multiband resonator is that its four resonant frequencies can be adjusted by changing the corresponding main branches, respectively. In this section, we numerically discuss the tuning effects of the multiband metamaterial by altering each main branch. Firstly, by changing the length of main branch $a$ from $4.5 \mathrm{~mm}$ to $3.7 \mathrm{~mm}$ by step of $0.2 \mathrm{~mm}$, and the simulated transmission results are presented in Figure 4(a). It clearly shows that the first transmission dip shifts from $10.85 \mathrm{GHz}$ towards the higher frequencies $11.86 \mathrm{GHz}$ and in the condition of $a=3.7 \mathrm{~mm}$ the first transmission dip disappeared. The second transmission reveals a slight blue shift, and the last two transmission dips 

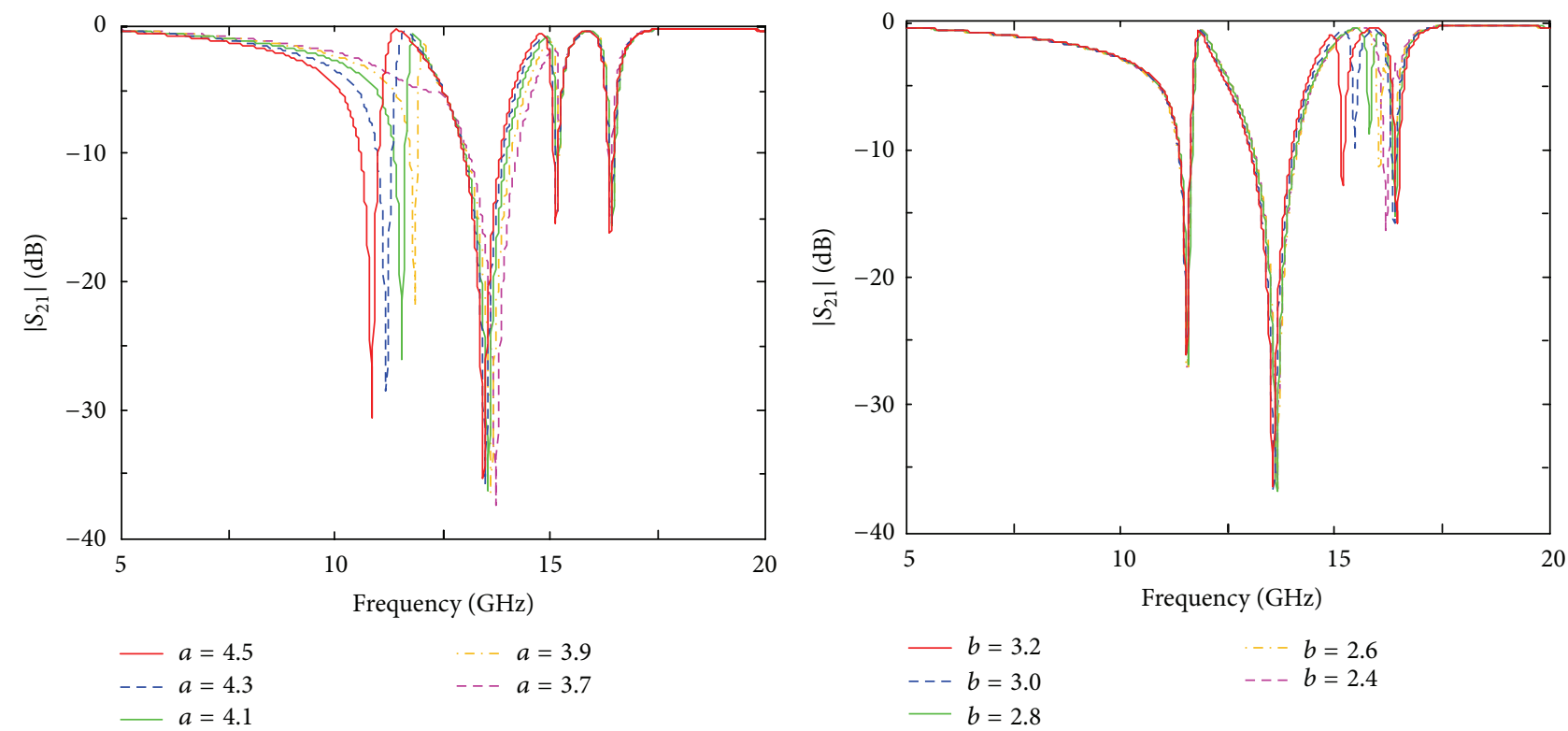

(a)

(b)

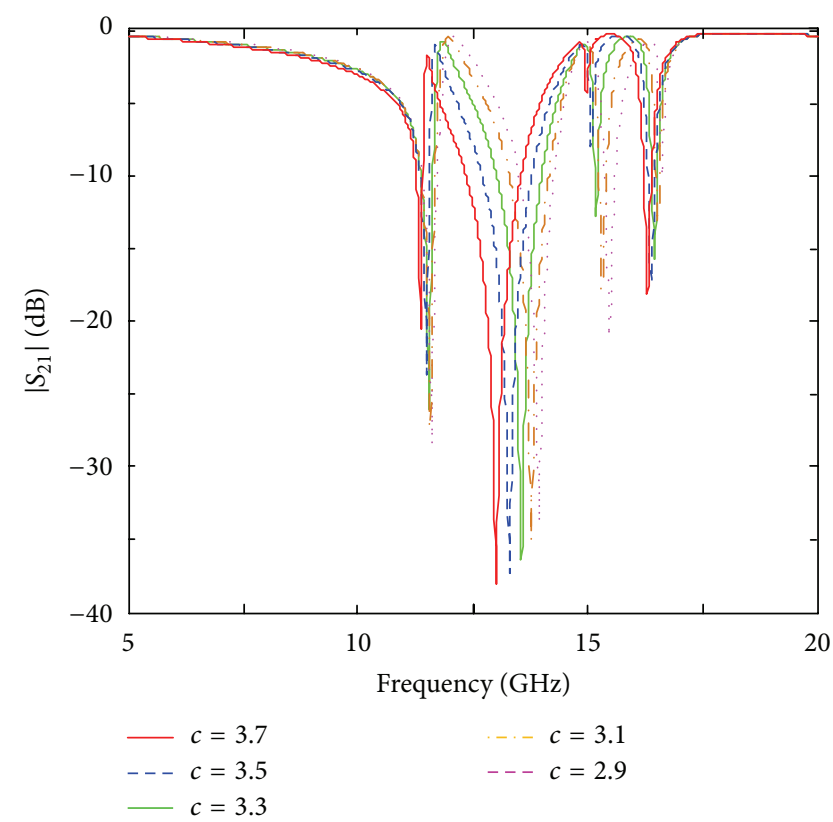

(c)

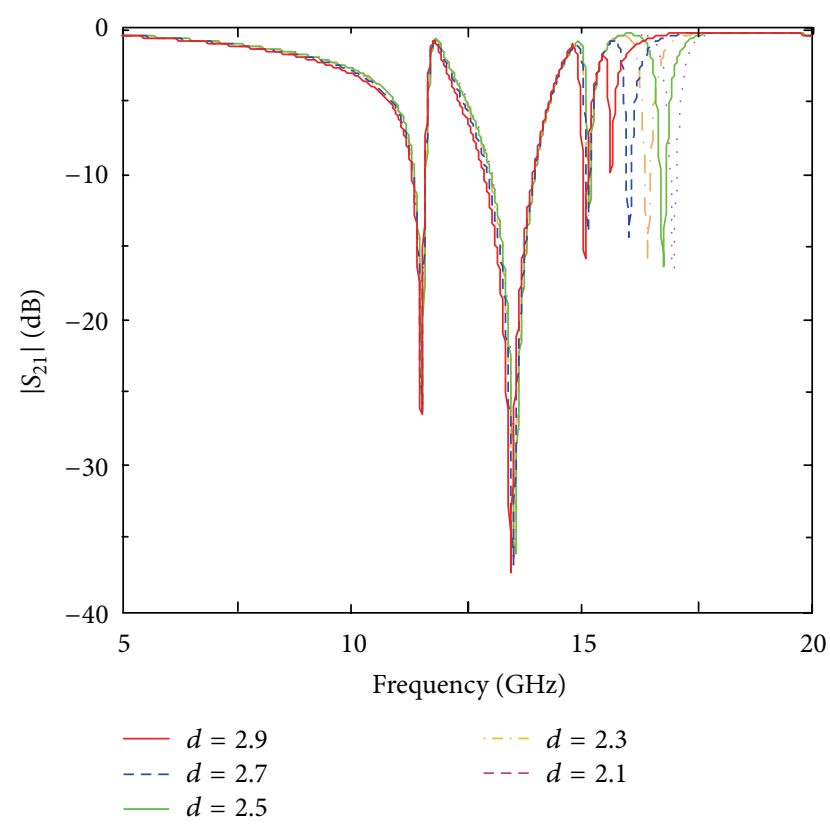

(d)

FIGURE 4: Simulated transmission properties of the proposed multiband metamaterial in different lengths for the four main branches (color online).

cannot be affected significantly. This means that the first transmission dip can be decided by the longest main branch with length $a$.

Secondly, when the length of another main branch $b$ was shortened from $3.2 \mathrm{~mm}$ to $2.4 \mathrm{~mm}$, the simulated tuning characteristics of the transmission results of the multiband metamaterial can be found in Figure 4(b). It is seen that the third transmission dip blue-shifts from $15.22 \mathrm{GHz}$ to 16.22 $\mathrm{GHz}$ and the other three transmission dips do not change. Therefore, this branch mainly contributes to the control of the third transmission dip.

By using the same method, we obtain the other two tuning properties for the second and forth transmission dips by changing the lengths of the main branches $c$ and $d$ from $3.7 \mathrm{~mm}$ to $2.9 \mathrm{~mm}$ and from $2.9 \mathrm{~mm}$ to $2.1 \mathrm{~mm}$, respectively. The numerical results are shown in Figures $4(\mathrm{c})$ and $4(\mathrm{~d})$, and it can be found that the corresponding transmission dips blue-shift from $13.02 \mathrm{GHz}$ to $13.95 \mathrm{GHz}$ and from $15.67 \mathrm{GHz}$ 


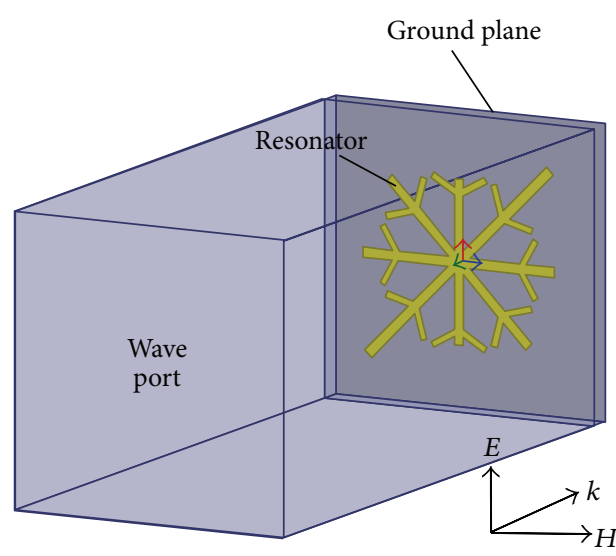

FIGURE 5: Numerical simulation setup of the metamaterial absorber composed of the multibranch resonator (color online).

to $17.03 \mathrm{GHz}$, respectively. Also, in each tuning condition, the other three transmission dips cannot be affected significantly.

These tuning properties provide a simple way to control the four operating frequencies and keep the whole period space unchanged. So it possesses the advantages in design of practical double negative metamaterials or metamaterial absorbers. In next section, we will design a multiband planar metamaterial absorber by using the previously discussed multibranch electric resonator.

\section{Multiband Absorber}

To construct the multiband metamaterial absorber, we just add another full-size copper plane with thickness of $0.017 \mathrm{~mm}$ on the other side of the dielectric substrate, with the similar method as shown in [20]. And in the numerical simulation setup, due to the full-size ground plane, there is not any EM wave which can be transmitted through the metamaterial absorber. So only one wave port is needed in simulations as shown in Figure 5.

In simulations, the other dimensional parameters of the metal resonator and dielectric substrate (except the thickness of the substrate) have the same values as mentioned in previous section. The absorptivity can be defined as $A(\omega)=$ $1-R(\omega)=1-\left|S_{11}\right|^{2}$ due to the ground plane, where the $R(\omega)$ is the simulated reflectivity. As analyzed in [20], the critical parameters to achieve the higher absorptivity are the thickness of the substrate, so in this paper, it is needed to optimize the value of the substrate thickness.

After optimizations, we get the near-equal four absorption peaks by setting the thickness of the substrate as $0.85 \mathrm{~mm}$. The simulated absorptivity property of the multiband metamaterial absorber composed of the proposed multibranch resonator is illustrated in Figure 6. It clearly shows that there are four absorption peaks located at $9.91 \mathrm{GHz}, 11.08 \mathrm{GHz}, 14.26 \mathrm{GHz}$, and $15.76 \mathrm{GHz}$, respectively. However, the four absorption peaks are correspondingly lower than the transmission dips as shown in Figure 2. This is due to fact that the added ground plane would increase

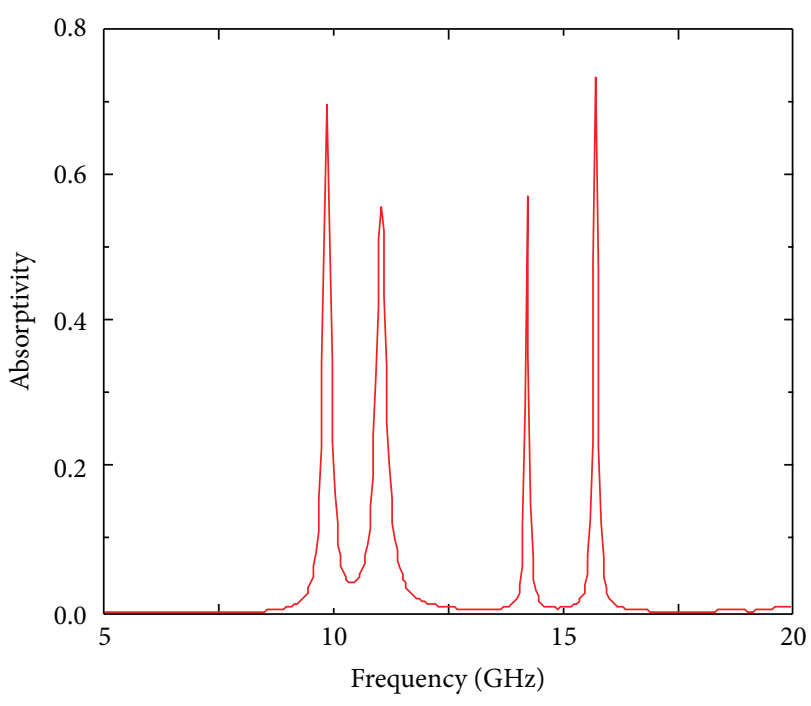

FIGURE 6: Simulated absorptivity characteristics of the proposed multiband metamaterial absorber (color online).

the inductances and capacitances between the metallic resonance and such ground plane, which would significantly reduce the resonance frequencies. Moreover, the surface current distributions in the multibranch resonator and ground plane at each absorbing peaks are presented in Figure 7 to understand the absorbing mechanism of the proposed multiband metamaterial absorber. As can be seen, this kind of metamaterial absorber exhibits typical electric resonance and magnetic resonance (due to the antiparallel currents between the resonator and ground plane) characteristics at all the four absorption peaks, similar to the conventional metamaterial absorbers [20].

Moreover, the absorption strength at the four absorbing frequencies of the proposed multiband metamaterial absorber can be enhanced separately and/or simultaneously by altering the thicknesses and the kinds (with different dielectric loss) of the substrate, the period space, and the kinds of the metal materials (not discussed in this paper). Some of the optimizing methods can be found in recently developed research paper such as in [20-22].

\section{Conclusion}

In this paper, we have demonstrated a novel kind of multiband negative permittivity metamaterial composed of multibranch electric resonator. The proposed metamaterial can exhibit four electric resonant frequencies, that resulted in four negative permittivity bands. Moreover, the four electric resonant frequencies can be tuned by changing the main branches. Also, we designed a multiband metamaterial absorber by using the proposed multibranch resonator and numerically demonstrated its absorptivities and surface current distributions. These results can enlarge the design categories of the band-enhanced metamaterials and the multiband applications. 

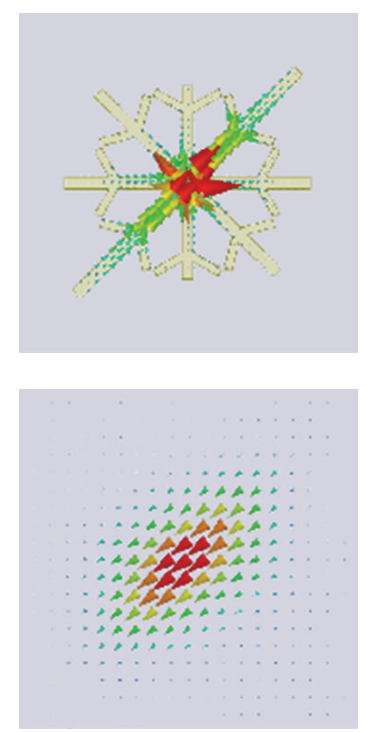

(a)
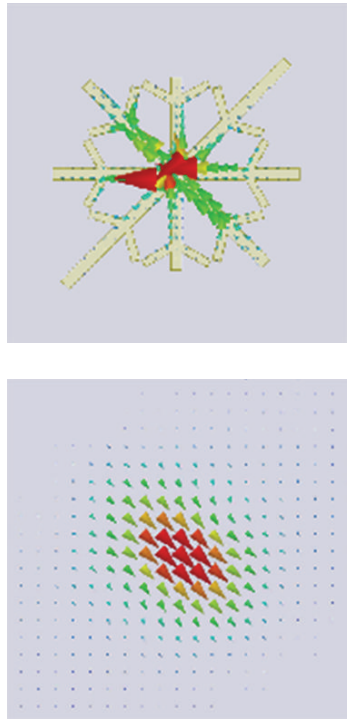

(b)
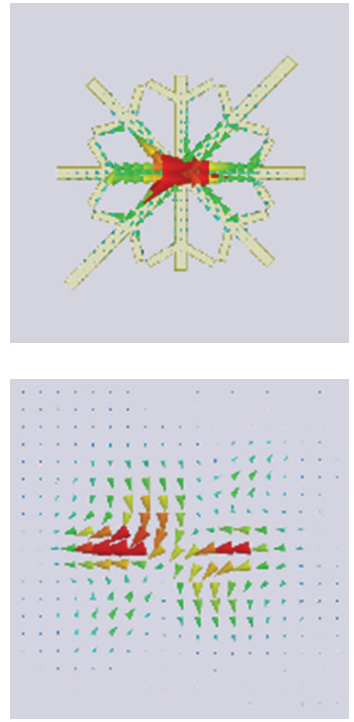

(c)
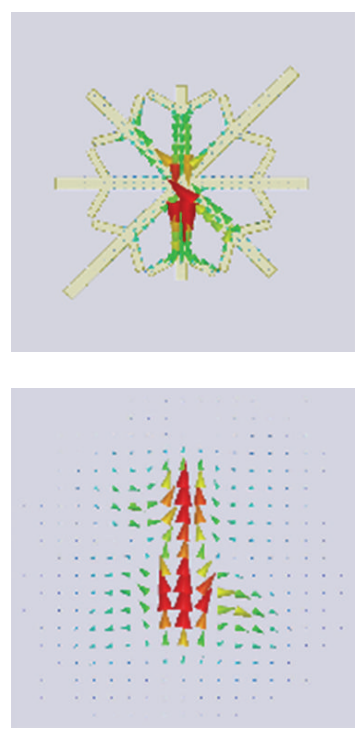

(d)

FiguRE 7: Surface current distributions in the resonator and the ground plane at the absorbing frequency: (a) $9.91 \mathrm{GHz}$, (b) $11.08 \mathrm{GHz}$, (c) 14.26 GHz, and (d) $15.76 \mathrm{GHz}$ (color online).

\section{Conflict of Interests}

The authors confirm that they have no conflict of interests.

\section{Acknowledgments}

This work was supported by Research Fund for the Doctoral Program of Higher Education of China (Grant no. 20110185110014). Yongjun Huang gratefully acknowledges the Scholarship Award for Excellent Doctoral Student granted by Ministry of Education of China.

\section{References}

[1] R. A. Shelby, D. R. Smith, and S. Schultz, "Experimental verification of a negative index of refraction," Science, vol. 292, no. 5514, pp. 77-79, 2001.

[2] H. Liang, Y. Shao, J. Zhou, B. A. Malomed, and G. Kurizki, "Conditions of perfect imaging in negative refraction materials with gain," Advances in OptoElectronics, vol. 2012, Article ID 347875, 5 pages, 2012.

[3] A. Grbic and G. V. Eleftheriades, "Experimental verification of backward-wave radiation from a negative refractive index metamaterial," Journal of Applied Physics, vol. 92, no. 10, pp. 5930-5935, 2002.

[4] G. V. Eleftheriades and K. G. Balmain, Negative-Refraction Metamaterials, John Wiley \& Sons, Hoboken, NJ, USA, 2005.

[5] L. M. Si, W. Zhu, and H. J. Sun, "A compact, planar, CPW-fed metamaterial-inspired dual-band antenna," IEEE Antennas and Wireless Propagation Letters, vol. 12, pp. 305-308, 2013.

[6] I. D. Rukhlenko, M. Premaratne, and G. P. Agrawal, "Plasmonic modes of metamaterial-based slot waveguides," Advances in OptoElectronics, vol. 2012, Article ID 907183, 5 pages, 2012.

[7] Y. Huang, G. Wen, J. Li, P. Wang, and Y. Sun, "Wide-angle and polarization-independent metamaterial absorber based on snowflake-shaped configuration," Journal of Electromagnetic Waves and Applications, vol. 27, no. 5, pp. 552-559, 2013.

[8] W. Zhu, M. Premaratne, and Y. Huang, "Hiding inside an arbitrarily shaped metal pit using homogeneous metamaterials," Journal of Electromagnetic Waves and Applications, vol. 26, pp. 2315-2322, 2012.

[9] W. Kan, B. Liang, X. Zhu et al., "Acoustic illusion near boundaries of arbitrary curved geometry," Scientific Reports, vol. 3, Article ID 1427, 6 pages, 2013.

[10] W. Zhu, X. Zhao, and B. Gong, "Left-handed metamaterials based on a leaf-shaped configuration," Journal of Applied Physics, vol. 109, no. 9, Article ID 093504, 2011.

[11] C. Zaichun, M. Rahmani, G. Yandong, C. T. Chong, and H. Minghui, "Realization of variable three-dimensional terahertz metamaterial tubes for passive resonance tunability," Advanced Materials, vol. 24, pp. OP143-OP147, 2012.

[12] S. Schwaiger, A. Rottler, and S. Mendach, "Rolled-up metamaterials," Advances in OptoElectronics, vol. 2012, Article ID 782864, 10 pages, 2012.

[13] E. P. Furlani, H. S. Jee, H. S. Oh, A. Baev, and P. N. Prasad, "Laser writing of multiscale chiral polymer metamaterials," Advances in OptoElectronics, vol. 2012, Article ID 861569, 7 pages, 2012.

[14] J. Zhong, Y. Huang, G. Wen, H. Sun, O. Gordon, and W. Zhu, "Dual-band negative permittivity metamaterial based on cross circular loop resonator with shorting stubs," IEEE Antennas and Wireless Propagation Letters, vol. 11, pp. 803-806, 2012.

[15] M. Li, Z. Wen, J. Fu et al., "Composite metamaterials with dualband magnetic resonances in the terahertz frequency regime," Journal of Physics D, vol. 42, no. 11, Article ID 115420, 4 pages, 2009.

[16] W. Zhu, X. Zhao, and J. Guo, "Multibands of negative refractive indexes in the left-handed metamaterials with multiple dendritic structures," Applied Physics Letters, vol. 92, no. 24, Article ID 241116, 3 pages, 2008.

[17] Y.-J. Huang, G.-J. Wen, T.-Q. Li, J. L.-W. Li, and K. Xie, “Design and characterization of tunable terahertz metamaterials with 
broad bandwidth and low loss," IEEE Antennas and Wireless Propagation Letters, vol. 11, pp. 264-267, 2012.

[18] Y. J. Huang, G. J. Wen, Y. J. Yang, and K. Xie, “Tunable dual-band ferrite-based metamaterials with dual negative refractions," Applied Physics A, vol. 106, no. 1, pp. 79-86, 2012.

[19] J. Zhong, F. Wang, G. Wen et al., “Tunable triple-band negative permeability metamaterial consisting of single-loop resonators and ferrite," Journal of Electromagnetic Waves and Applications, vol. 27, no. 3, pp. 267-275, 2013.

[20] J. Zhong, Y. Huang, G. Wen, H. Sun, P. Wang, and O. Gordon, "Single-/dual-band metamaterial absorber based on crosscircular-loop resonator with shorted stubs," Applied Physics A, vol. 108, pp. 329-335, 2012.

[21] W. Zhu, Y. Huang, I. D. Rukhlenko, G. Wen, and M. Premaratne, "Configurable metamaterial absorber with pseudo wideband spectrum,” Optics Express, vol. 20, no. 6, pp. 6616-6621, 2012.

[22] F. Ding, Y. Cui, X. Ge, Y. Jin, and S. He, "Ultra-broadband microwave metamaterial absorber," Applied Physics Letters, vol. 100, no. 10, Article ID 103506, 3 pages, 2012.

[23] Y. Cui, K. H. Fung, J. Xu et al., "Ultrabroadband light absorption by a sawtooth anisotropic metamaterial slab," Nano Letters, vol. 12, no. 3, pp. 1443-1447, 2012.

[24] D. Schurig, J. J. Mock, and D. R. Smith, "Electric-field-coupled resonators for negative permittivity metamaterials," Applied Physics Letters, vol. 88, no. 4, Article ID 041109, pp. 1-3, 2006.

[25] W. J. Padilla, M. T. Aronsson, C. Highstrete, M. Lee, A. J. Taylor, and R. D. Averitt, "Electrically resonant terahertz metamaterials: theoretical and experimental investigations," Physical Review B, vol. 75, no. 4, Article ID 041102, 2007.

[26] D. R. Smith, D. C. Vier, T. Koschny, and C. M. Soukoulis, "Electromagnetic parameter retrieval from inhomogeneous metamaterials," Physical Review E, vol. 71, no. 3, Article ID 036617, 2005.

[27] R. Marqués, F. Medinaand, and R. Rafii-El-Idrissi, "Role of bianisotropy in negative permeability and left handed metamaterials," Physical Review B, vol. 65, Article ID 144441, 6 pages, 2002. 

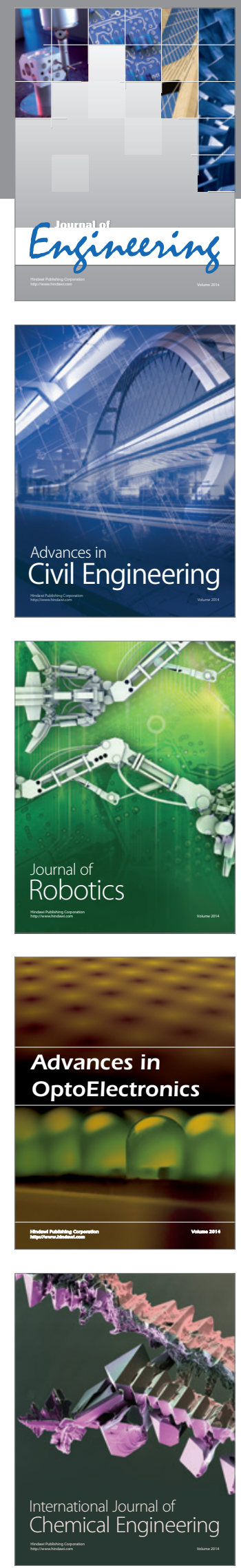

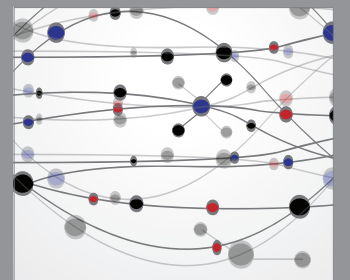

The Scientific World Journal
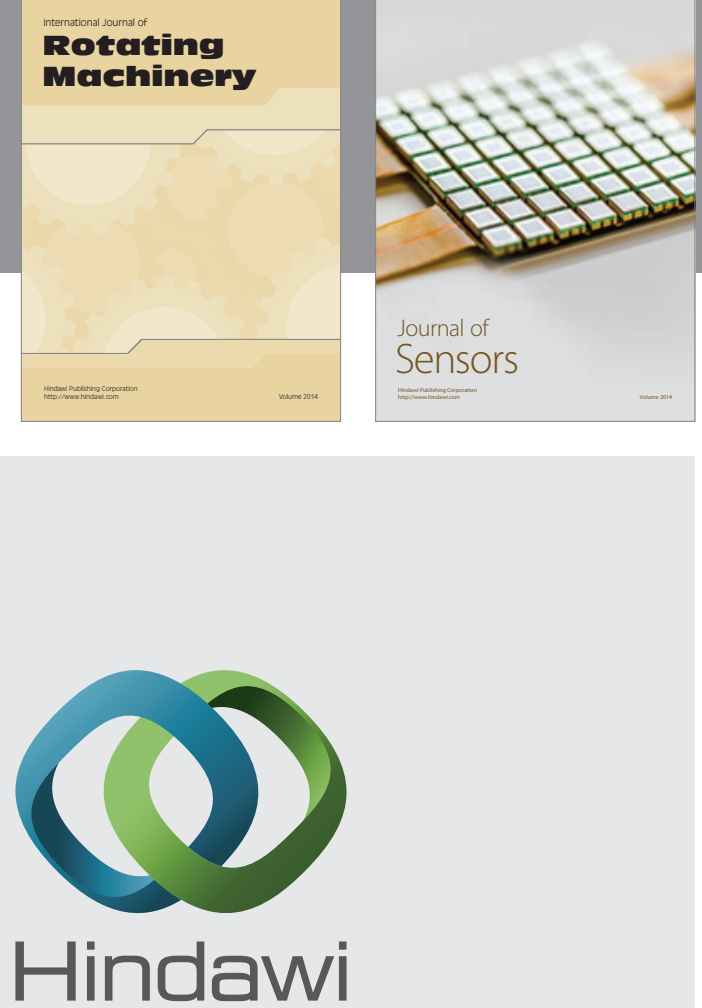

Submit your manuscripts at http://www.hindawi.com
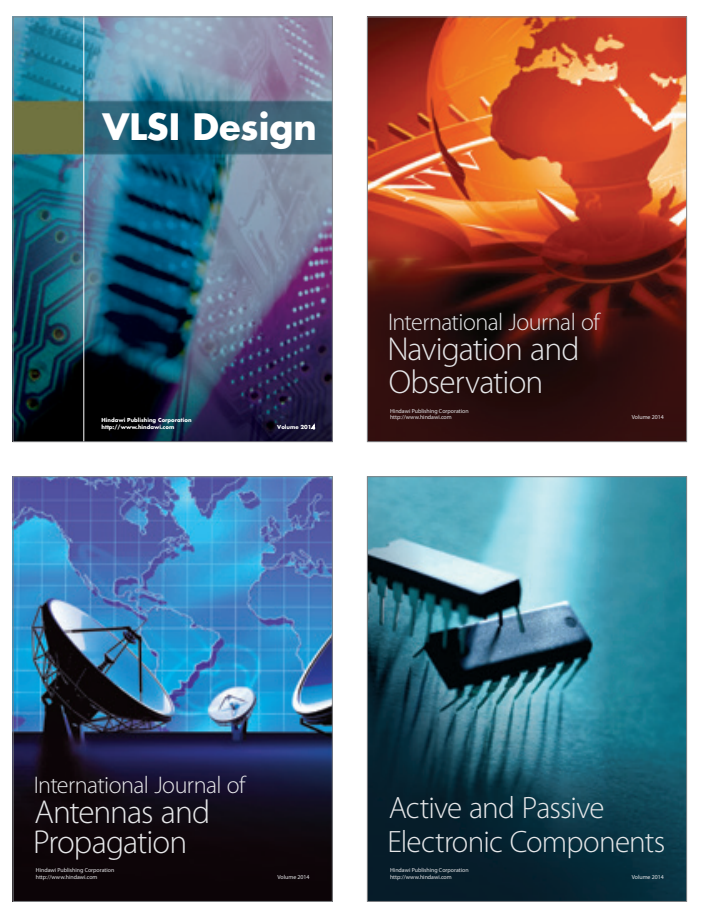
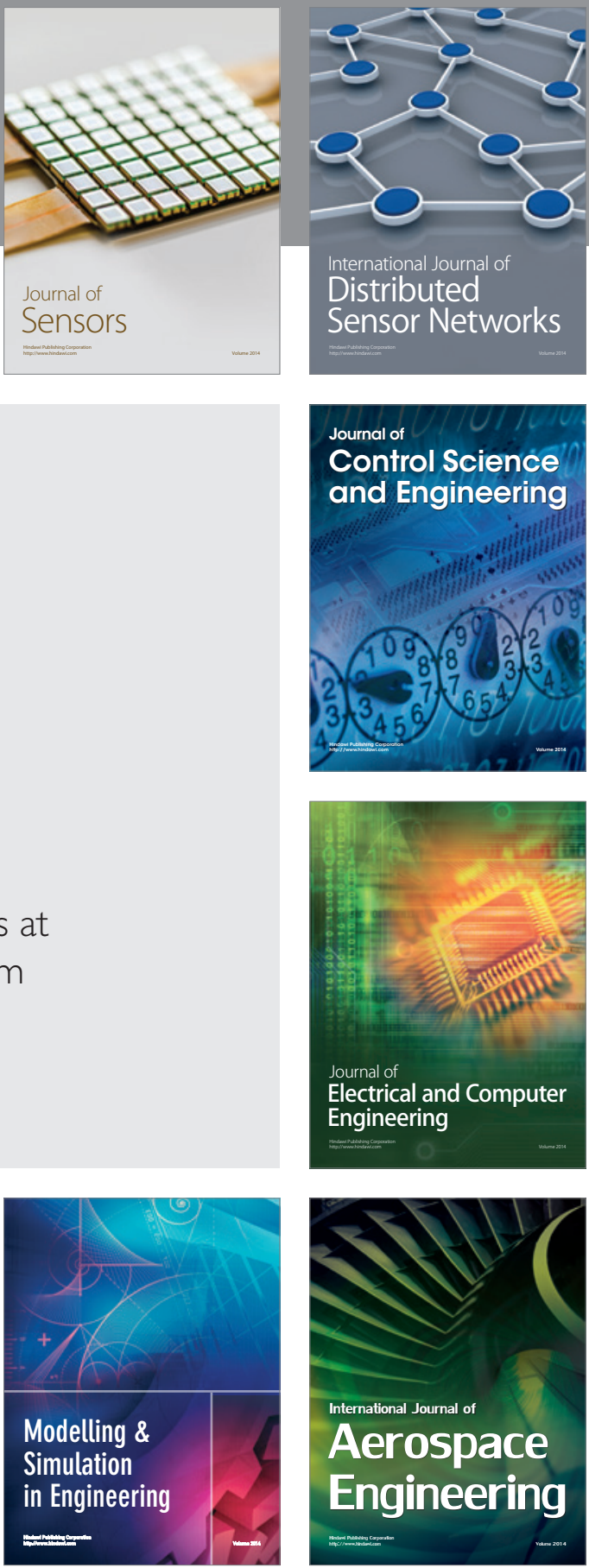

Journal of

Control Science

and Engineering
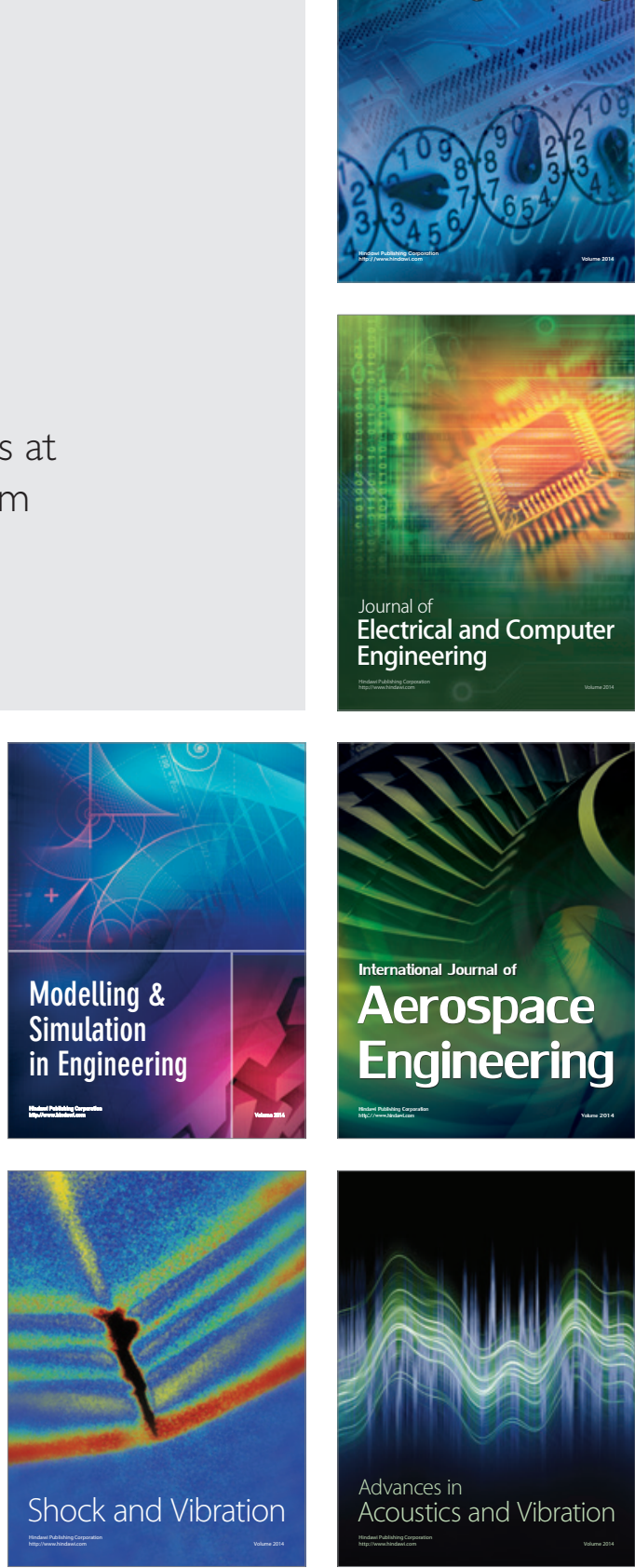